\title{
Novel norovirus recombinants and Gll.4 sub-lineages associated with outbreaks between 2006 and 2010 in Belgium
}

Elisabeth Mathijs ${ }^{1 \dagger}$, Sarah Denayer ${ }^{3 \dagger}$, Leonor Palmeira ${ }^{2}$, Nadine Botteldoorn ${ }^{3}$, Alexandra Scipioni ${ }^{1}$, Alain Vanderplasschen ${ }^{2}$, Etienne Thiry ${ }^{1 *}$ and Katelijne Dierick ${ }^{3}$

\begin{abstract}
Background: Noroviruses (NoVs) are an important cause of acute gastroenteritis in humans worldwide. To gain insight into the epidemiologic patterns of NoV outbreaks and to determine the genetic variation of NoVs strains circulating in Belgium, stool samples originating from patients infected with NoVs in foodborne outbreak investigations were analysed between December 2006 and December 2010.

Results: NoVs were found responsible of $11.8 \%$ of all suspected foodborne outbreaks reported in the last 4 years and the number of NoV outbreaks reported increased along the years representing more than $30 \%$ of all foodborne outbreaks in 2010. Genogroup II outbreaks largely predominated and represented more than 90\% of all outbreaks. Phylogenetic analyses were performed with 63 NoV-positive samples for the partial polymerase $(\mathrm{N}=45)$ and/or capsid gene $(N=35)$ sequences. For 12 samples, sequences covering the ORF1-ORF2 junction were obtained. A variety of genotypes was found among genogroups I and II; GII.4 was predominant followed in order of importance by GII.2, GII.7, GII.13, GI.4 and GI.7. In the study period, GIl.4 NoVs variants 2006a, 2006b, 2007, 2008 and 2010 were identified. Moreover, phylogenetic analyses identified different recombinant NoV strains that were further characterised as intergenotype (GIl.e/GIl.4 2007, GIl.e/GIl.3 and GIl.g/GII.1) and intersub-genotype (GIl.4 2006b/GIl.4 2007 and GII.4 2010/GIl.4 2010b) recombinants.

Conclusions: NoVs circulating in the last 4 years in Belgium showed remarkable genetic diversity either by smallscale mutations or genetic recombination. In this period, Gll.4 2006b was successfully displaced by the Gll.4 2010 subtype, and previously reported epidemic Gll.b recombinants seemed to have been superseded by Gll.e recombinants in 2009 and Gll.g recombinants in 2010. This study showed that the emergence of novel Gll.4 variants together with novel Gll recombinants could lead to an explosion in NoV outbreaks, likewise to what was observed in 2008 and 2010. Among recombinants detected in this study, two hitherto unreported strains GIl.e/GIl.3 and Gll.g/Gll.1 were characterised. Surveillance will remain important to monitor contemporaneously circulating strains in order to adapt preventive and curative strategies.
\end{abstract}

\footnotetext{
* Correspondence: etienne.thiry@ulg.ac.be

† Contributed equally

'Department of Infectious and Parasitic Diseases, Veterinary Virology and Animal Viral Diseases, Faculty of Veterinary Medicine, University of Liege, Liege, Belgium

Full list of author information is available at the end of the article
} 


\section{Background}

Noroviruses (NoVs) are on the rise as a causative agent of gastroenteritis in humans of all ages and are responsible for approximately $90 \%$ of epidemic non-bacterial outbreaks of gastroenteritis in the world. Transmission can occur through direct contact with shedding persons, contaminated food, sewage-contaminated water, contaminated aerosols and environmental contamination [1]. Food-borne transmissions have been estimated to account for $14 \%$ of infections due to NoV [2]. The major issue with NoV infections is their frequent occurrence in large outbreaks in community settings such as hospitals and nursing homes $[3,4]$.

The genus Norovirus belongs to the family Caliciviridae along with 4 other genera (Vesivirus, Lagovirus, Sapovirus and Nebovirus). NoVs are non-enveloped viruses with a single-stranded, positive-sense, polyadenylated RNA genome of about 7500 nucleotides (nt) in length [5]. Three overlapping ORFs encode the nonstructural (ORF1) and structural (ORF2 and ORF3) viral proteins. The ORF1-encoded polyprotein is cleaved further by the viral proteinase into six mature products including the genome-linked virus protein (VPg), the proteinase and the highly conserved RNA-dependent RNA-polymerase [6]. Due to its high conservation, the polymerase has been a widely used target for molecular detection assays [7]. ORF 2 encodes the major capsid protein (VP1) that contains an N-terminal arm, a shell or S-domain and a protrusion or P-domain. The Pdomain is divided into 2 sub-domains called P1 and P2, the latter corresponding to the most variable region of the capsid. ORF3 encodes for a small minor structural protein of the virion [8].

NoVs are genetically highly diverse and are divided into five genogroups (GI-V) which are further subdivided into at least 29 genotypes based on genetic differences in the capsid gene [9-11]. GI and GII NoVs are responsible for most human infections. The absence of a regular cell-culture system or small-animal model for human NoVs led to the development of molecular tools to study the epidemiology of NoVs. GII.4 has been by far the most detected genotype being responsible for 60 to $70 \%$ of the outbreaks globally reported to the Foodborne Viruses in Europe (FBVE) network between 1990 and 2008 [12]. During this same period, 4 large pandemics (1995-1996, 2002, 2004-2005 and 2006) have been identified corresponding each time to the emergence of one or two new variants of the GII.4 lineage and the displacement of the previously predominant circulating ones [13,14]. Frequently reported as second most prevalent NoVs after GII.4 strains, are GII.b recombinants [15]. Since the first detection in France in August 2000, they have been involved in outbreaks across the globe thereby demonstrating their spreading capacity among human populations $[16,17]$. Along with small-scale mutations, recombination events seem to contribute to the genetic variability between NoVs. This phenomenon was identified by incongruent clustering of different regions of the genome in phylogenetic analyses for NoVs from all 5 genogroups [18-20]. Based upon predictive models, the majority of NoV recombinants have breakpoints located either within or close to the ORF1-ORF2 junction. Consequently, this region has been suggested to constitute a recombination hotspot in NoVs [21].

To gain insight into the epidemiologic patterns of NoV outbreaks and to determine the genetic variation of NoVs strains circulating in Belgium, we analyzed stool samples originating from patients infected with NoVs in foodborne outbreak investigations conducted by the Belgian Scientific Institute of Public Health (IPH). These studies involved predominantly large outbreaks occurring in community settings between December 2006 and December 2010.

\section{Methods \\ Definitions}

At European level a food-borne outbreak (FBO) is defined as an incidence, observed under given circumstances, of two or more human cases of the same disease and/or infection; or a situation in which the observed number of human cases exceeds the expected number and where the cases are linked, or are probably linked, to the same food source (Directive 2003/99/EC, Article 2(d)). A human NoV infection is described as a case with the typical gastroenteritis symptoms as started between 12-24 $\mathrm{h}$ after infection, nausea, vomiting, diarrhoea and in some cases also slight fever along with the laboratory confirmation of the presence of NoVs in clinical samples.

\section{Sample collection and stool specimens}

Since 2005, the IPH harbors the National Reference Laboratory for foodborne outbreaks (NRL FBO) and it is responsible for collecting, centralizing the information, reporting and follow up of FBOs in Belgium. In case of an outbreak, stool samples were sent to the IPH by the Health Inspectors or clinical laboratories at hospitals. Food samples were sent to the NRL FBO by the local inspectors of the Federal Agency for Safety of the Food Chain (FASFC). Samples were stored refrigerated $\left(2-8^{\circ} \mathrm{C}\right)$ during transport and analyses started the day of arrival at the NRL FBO. In total, $164 \mathrm{NoV}$-positive stool specimens from cases of acute gastro-enteritis were collected during the 4-year period from December 2006 through December 2010. 


\section{Laboratory investigation: norovirus detection}

Stool suspensions (10\%, [weight/volume]) were prepared in phosphate buffered saline (PBS). One hundred microliters supernatant was collected after centrifugation for 5 minutes at $13000 \mathrm{rpm}$ (Eppendorf 5415D, Rotselaar, Belgium). RNA was extracted using the RNeasy Mini Kit (Qiagen, Leusden, The Netherlands) following the manufacturer's instructions. NoV was extracted from foodstuff according to the protocol as previously described with slight modifications [22]. Briefly, $10 \mathrm{~g}$ of food was homogenized with $8 \mathrm{ml}$ TRIzol ${ }^{\circledR}$ reagent (Invitrogen, Merelbeke, Belgium) and shaken for $20 \mathrm{~min}$ at room temperature to allow contact. After centrifugation for 10 minutes $(13000 \mathrm{rpm})$ at $4^{\circ} \mathrm{C}$ (Eppendorf 5804R, Rotselaar, Belgium) the aqueous phase is transferred into a new tube for further concentration/purification. Two hundred microliters of chloroform is added for each $\mathrm{ml}$ of TRIzol ${ }^{\mathbb{B}}$ reagent used, and mixed for $15 \mathrm{~s}$ followed by $2-3 \mathrm{~min}$ settling before centrifugation $\left(13000 \mathrm{rpm}, 15 \mathrm{~min}, 4^{\circ} \mathrm{C}\right)$. One hundred microliters of the upper (aqueous) phase was used for RNA extraction using the RNeasy Mini Kit (Qiagen, Leusden, The Netherlands) following the manufacturer's instructions. The reverse transcription (RT) step was performed using the Transcriptor High Fidelity cDNA synthesis kit (Roche, Vilvoorde, Belgium) according to the manufacturer's instructions. Real-Time PCR ( $\mathrm{qPCR}$ ) was performed using available primers and probes for the detection of the majority of the human GI (QNIF4, CGCTG GATGCGNTTCCAT; NV1LCR, CCTTAGACGCCATCATCATTTAC; NV1LCpr, FAMTGGACAGGAGAYCGCRATCT-TAMRA) and GII (QNIF2, ATG TTCAGRTGGATGAGRTTCTCWGA; COG2R, TCG ACGCCATCTTCATTCACA; QNIFS, FAM-AGCA CGTGGGAGGGCGATCG-TAMRA) NoV strains, recommanded by the CEN/TC/WG6/TAG4 research group [23,24]. Double stranded DNA (plasmid DNA GI and plasmid DNA GII) was used as positive control of the qPCR at a concentration of 2500 copies per reaction [25].

\section{RNA preparation, genomic amplification for genotyping}

For all samples tested positive for norovirus by RTqPCR, viral RNA was re-extracted from $140 \mu \mathrm{l}$ sample supernatant with the QIAamp viral RNA mini kit (Qiagen, Leusden, The Netherlands) according to the manufacturer's instructions. RNA extracts were stored at $-80^{\circ}$ $\mathrm{C}$ before use. For genotyping, published primers were used for the amplification and sequencing of the 3' of the polymerase gene (region A in ORF1), the 5 ' end of the capsid gene (region $\mathrm{C}$ in ORF2) and the nearly full length major capsid gene $[7,26,27]$ (Table 1 and 2). First-stranded cDNA was generated by an iScript cDNA Synthesis kit (Bio-Rad, Nazareth, Belgium). PCRs were carried out on $4 \mu \mathrm{l}$ cDNA in $50 \mu \mathrm{l}$ nuclease-free water containing $300 \mathrm{nM}$ of both forward and reverse primers, $0.1 \mathrm{mM}$ dNTPs, 2.5\% DMSO, $20 \mathrm{mM}$ Tris/ $\mathrm{HCl}, 10 \mathrm{mM}$ (NH4)2SO4, $10 \mathrm{mM} \mathrm{KCl,} 2 \mathrm{mM} \mathrm{MgSO} 4,0.1 \%$ Triton $\mathrm{X}-100$ and $1 \mathrm{U}$ Taq DNA Polymerase (New England Biolabs, Leusden, The Netherlands). The nearly complete capsid gene and ORF1-ORF2 junction were amplified using Iproof High Fidelity polymerase (Biorad, Nazareth, Belgium). Sequences covering the overlap between ORF1 and ORF2 were amplified when phylogenetic analyses indicated incongruent clustering for the partial sequences of the polymerase and the capsid genes for the same samples. After amplification, the amplicons were visualized by electrophoresis and purified by using either a standard ethanol precipitation protocol or the QIAquick PCR purification kit (Qiagen, Leusden, The Netherlands) according to instructions given by the manufacturer. Direct sequencing of PCR products was carried out by GATC Biotech sequencing facilities (Konstanz, Germany) in both directions using an ABI 3730xl DNA Analyzer (Applied Biosystems, Lennik, Belgium).

\section{Molecular typing, phylogenetic analysis and recombination study}

All sequences were typed with the genotyping tool for NoVs, National Institute of Public Health and the Environment, The Netherlands at http://www.rivm.nl. Database searches for related sequences were conducted using BLAST. Reference strains for phylogenetic analysis were selected from the Norovirus genotyping tool and are given in the Additional file 1 Table S1. All sequences were aligned at the protein level using the MUSCLE algorithm [28] to produce more robust alignments and were back-translated into nucleic acid sequences for subsequent analyses. Phylogenetic trees were inferred on the previously aligned sequences under a maximum-likelihood framework using PhyML [29] under a GTR evolutionary model [30] with optimized invariable sites and a discrete gamma model with 4classes of optimized rates to model the variability of substitution rates across sites [31]. Branch support was inferred using the Shimodaira-Hasegawa-like non-parametric procedure [32]. To accommodate for weak phylogenetic signal, a thorough exploration of the tree space was made through topological rearrangements using a combination of Nearest Neighbor Interchange and Subtree Pruning and Regrafting topology search methods as implemented in PhyML [33,34]. Finally, for visualization purposes, the trees were then arbitrarily rooted.

\section{Nucleotide sequence accession numbers}

Accession numbers for sequences obtained for partial polymerase gene, partial capsid gene and/or partial 
Table 1 Characteristics of genotyped norovirus-positive clinical samples

\begin{tabular}{|c|c|c|c|c|c|c|}
\hline Year & Sample Name & Outbreak code & Outbreak localisation & Date of first symptoms & Sequenced region & Genotyping result \\
\hline \multirow[t]{3}{*}{2006} & IPH55 & HAl0016 & Hainaut & $22 / 12 / 2006$ & A & Gll.4 2006b \\
\hline & IPH57 & & & & $A+C$ & Gll.4 2006b \\
\hline & IPH59 & & & & A & Gll.4 2006b \\
\hline \multirow[t]{5}{*}{2007} & IPH472 & - & Namur & $10 / 04 / 2007$ & $A+C$ & Gll.4 2006a \\
\hline & IPH473 & & & & $A+C$ & Gll.4 2006a \\
\hline & IPH474 & & & & $A+C$ & Gll.4 2006a \\
\hline & IPH475 & & & & $A+C$ & Gll.4 2006a \\
\hline & IPH477 & & & & $A+C$ & Gll.4 2006a \\
\hline \multirow[t]{14}{*}{2008} & $\mathrm{IPH} 2159$ & 08VG06 & Limburg & $30 / 09 / 2008$ & A & Gll.2 \\
\hline & IPH2161 & & & & Junction & Gll.2 \\
\hline & $\mathrm{IPH} 2162$ & & & & Junction & Gll.2 \\
\hline & $\mathrm{IPH} 2204$ & 08VG01 & Flemish Brabant & $1 / 10 / 2008$ & Junction & GII.4 2008 \\
\hline & IPH2206 & & & & Junction & GIl.4 2008 \\
\hline & $\mathrm{PH} 2252$ & VBR008 & Flemish Brabant & $30 / 09 / 2008$ & C & Gl.4 \\
\hline & $\mathrm{IPH} 2255$ & & & & Junction & $\mathrm{Gl} .4$ \\
\hline & $\mathrm{PH} 2700$ & 08VG07 & East Flanders & $14 / 11 / 2008$ & $A+C$ & Gll.4 2006b/GIl.4 2007 \\
\hline & IPH2839 & OVL009 & East Flanders & $26 / 11 / 2008$ & $\begin{array}{c}\text { Junction } \\
\text { A }\end{array}$ & $\begin{array}{l}\text { Gll.e/Gll.4 2007 } \\
\text { Gll.4 2006b/- }\end{array}$ \\
\hline & IPH3007 & ANT005 & Antwerp & $2 / 12 / 2008$ & $A+C$ & Gll.7 \\
\hline & IPH3059 & 08VG08 & Limburg & $12 / 12 / 2008$ & A & Gll.4 2006b \\
\hline & IPH3060 & & & & $\mathrm{A}+\mathrm{C}$ & Gll.4 2006b \\
\hline & IPH3064 & & & & A & Gll.4 2006b \\
\hline & IPH3069 & & & & $A+C$ & Gll.e/Gll.4 2007 \\
\hline \multirow[t]{8}{*}{2009} & $\mathrm{IPH} 143$ & 09VG2 & Limburg & $6 / 02 / 2009$ & Junction & Gll.e/GIl.4 2007 \\
\hline & IPH608 & 09VG3 & Limburg & 22/03/2009 & Junction & Gll.4 2006b \\
\hline & $\mathrm{PH} 2171$ & 09VG4 & Limburg & 05/05/2009 & $A+C$ & Gll.e/Gll.3 \\
\hline & IPH2172 & & & & Junction & Gll.e/Gll.3 \\
\hline & $\mathrm{IPH} 2174$ & & & & $A+C$ & Gll.e/Gll.3 \\
\hline & $\mathrm{IPH} 2175$ & & & & Junction & Gll.e/GIl.3 \\
\hline & $\mathrm{IPH} 2176$ & & & & $A+C$ & Gll.e/Gll.3 \\
\hline & $\mathrm{PH} 3075$ & ANT011 & Antwerp & $8 / 12 / 2009$ & A & Gll.4 2010/- \\
\hline \multirow[t]{18}{*}{2010} & IPH159 & 10VG1 & Limburg & $27 / 01 / 2010$ & A & Gll.4 2010/- \\
\hline & $\mathrm{IPH} 161$ & & & & $A+C$ & Gll.4 2010 \\
\hline & $\mathrm{IPH} 163$ & LIM001 & Limburg & 27/01/2010 & Junction & Gll.g/GIl.1 \\
\hline & $\mathrm{IPH} 164$ & & & & $A+C$ & Gll.g/Gll.1 \\
\hline & $\mathrm{IPH} 165$ & 10VG2 & Limburg & $1 / 02 / 2010$ & $A+C$ & Gll.4 2010/GIl.4 2010b \\
\hline & IPH167 & & & & A & $\mathrm{Gl} .7 /-$ \\
\hline & $\mathrm{IPH} 251$ & & & & $A+C$ & Gll.4 2010/Gll.4 2010b \\
\hline & $\mathrm{IPH} 294$ & 10VG3 & Brussels & $2 / 02 / 2010$ & $A+C$ & Gll.4 2010/GIl.4 2010b \\
\hline & IPH296 & & & & $\mathrm{A}+\mathrm{C}$ & Gll.4 2010/GIl.4 2010b \\
\hline & IPH297 & & & & $A+C$ & Gll.4 2010/GIl.4 2010b \\
\hline & IPH298 & & & & $A+C$ & Gll.4 2010/GIl.4 2010b \\
\hline & $\mathrm{IPH} 301$ & & & & $A+C$ & Gll.4 2010/Gll.4 2010b \\
\hline & $\mathrm{IPH} 310$ & 10VG9 & Antwerp & $17 / 02 / 2010$ & $C$ & $-/ G \| l .42010 b$ \\
\hline & IPH312 & & & & $\mathrm{A}+\mathrm{C}$ & Gll.4 2010/GIl.4 2010b \\
\hline & $\mathrm{IPH} 314$ & & & & $A+C$ & Gll.4 2010/Gll.4 2010b \\
\hline & $\mathrm{PH} 317$ & 10VG4 & Antwerp & $17 / 02 / 2010$ & $A+C$ & Gll.4 2010/GIl.4 2010b \\
\hline & $\mathrm{IPH} 505$ & 10VG5 & Antwerp & 04/03/2010 & A & Gll.g/- \\
\hline & IPH506 & 10CF1 & Namur & $04 / 03 / 2010$ & $A+C$ & Gll.4 2010/GIl.4 2010b \\
\hline
\end{tabular}


Table 1 Characteristics of genotyped norovirus-positive clinical samples (Continued)

\begin{tabular}{|c|c|c|c|c|c|}
\hline IPH671 & & & & $A+C$ & Gll.4 2010/Gll.4 2010b \\
\hline IPH748 & LIM003 & Limburg & 05/04/2010 & C & $-/ G I I .13$ \\
\hline IPH966 & LIM005 & Limburg & 08/05/2010 & $A+C$ & Gll.g/Gll.1 \\
\hline IPH967 & & & & $A+C$ & Gll.g/GIl.1 \\
\hline IPH968 & & & & A & Gll.g/- \\
\hline $\mathrm{PPH} 1010$ & ANT003 & Antwerp & $8 / 05 / 2010$ & $A+C$ & Gll.4 2010 \\
\hline $\mathrm{IPH} 1093$ & OVL005 & East Flanders & 19/05/2010 & A & Gll.7/- \\
\hline $\mathrm{PPH} 1143$ & ANT004 & Antwerp & $18 / 05 / 2010$ & $A+C$ & Gll.2 \\
\hline IPH1919 & VBR006 & Flemish Brabant & 17/07/2010 & $A+C$ & Gll.g/Gll.1 \\
\hline IPH1936 & & & & $A+C$ & Gll.g/Gll.1 \\
\hline $\mathrm{IPH} 1920$ & LIM007 & Limburg & $17 / 07 / 2010$ & C & $-/ G \| l .1$ \\
\hline $\mathrm{PPH} 2020$ & LIM008 & Limburg & $27 / 07 / 2010$ & $A+C$ & Gll.g/Gll.1 \\
\hline IPH2021 & & & & $A+C$ & Gll.g/Gll.1 \\
\hline IPH2O22 & & & & $A+C$ & Gll.g/Gll.1 \\
\hline IPH2O45 & & & & Junction & Gll.g/Gll.1 \\
\hline
\end{tabular}

A: partial polymerase gene; C: partial capsid gene; Junction: ORF1-ORF2 overlap

polymerase and capsid gene with the overlapping junction of the ORF1-ORF2 junction in this study are: [GenBank: EU794891-EU794894; EU794879-EU794883; JF697202-JF697293].

\section{Results}

Characterisation of NoV outbreaks in Belgium, 2006-2010 From December 2006 to December 2010, a total of 458 gastroenteritis outbreaks, suspected to be food-borne, were reported to the Belgian IPH (Additional file 2 Table S2). During this period, NoVs were involved in 54 $(11.8 \%)$ of the reported outbreaks and affected $34.5 \%$ of all patients reported ill. Classification of NoVs into genogroups GI and GII was possible for all samples tested positive by RT-qPCR but more accurate genotyping (genotype and sub-genotype) was only possible for over half of the NoV outbreaks (Additional file 2 Table S2).
Indeed, phylogenetic analyses were performed with 63 NoV-positive samples for the partial polymerase $(\mathrm{N}=$ $45)$ and/or capsid gene $(\mathrm{N}=35)$ sequences. For 12 samples, sequences covering the ORF1-ORF2 junction were obtained. Despite the fact that NoVs were detected in some food samples, no sequences could be obtained as amplification by conventional RT-PCR was unsuccessful. With the exception of 2009, the number of reported NoV outbreaks increased throughout the years; particularly in 2010 where NoV outbreaks represented more than $30 \%$ of the reported outbreaks. When the monthly distribution of NoV outbreaks over the 4-year study period was plotted, two peaks in NoV activity could be seen; one in the autumn-winter 2008/2009 and another from December 2009 to September 2010 (Figure 1A). In both cases, the increase in NoV activity coincided with the detection of novel NoV strains. From 2006 until

Table 2 Oligonucleotide primers used for genotyping

\begin{tabular}{|c|c|c|c|c|c|}
\hline Region & Name & Sequence $\left(5^{\prime} \rightarrow 3^{\prime}\right)^{a}$ & Sense & Position $^{\text {b }}$ & Reference \\
\hline A & 290d & GATTACTCCASSTGGGAYTCMAC & + & $4568-4590$ & {$[50]$} \\
\hline A & $289 d$ & TGACGATTTCATCATCMCCRTA & - & $4865-4886$ & \\
\hline A & $J V 12 Y$ & ATACCACTATGATGCAGAYTA & + & $4552-4572$ & [7] \\
\hline A & JV13I & TCATCATCACCATAGAAIGAG & - & $4858-4878$ & \\
\hline C & G1SKF & CTGCCCGAATTYGTAAATGA & + & $5342-5361$ & [26] \\
\hline C & G1SKR & CCAACCCARCCATTRTACA & - & $5652-5671$ & \\
\hline C & G2SKF & CNTGGGAGGGCGATCGCAA & + & $5046-5064^{c}$ & \\
\hline C & G2SKR & CCRCCNGCATRHCCRTTRTACAT & - & $5367-5389^{c}$ & \\
\hline Capsid & FW1 & GCGATCGCAATCTGGCTCCCAG & + & $5055-5076^{d}$ & {$[27]$} \\
\hline Capsid & RT5 & AGGTGYACATTATGACCAGTTC & - & $6795-6819^{d}$ & \\
\hline
\end{tabular}

${ }^{a}$ International Union of Biochemistry ambiguity codes: I, inosine; N, (A/C/T/G); H, (A/C/T); Y, (C/T); M, (A/C); R, (A/G); S, (C/G).

${ }^{b}$ Position in the norovirus genomic sequence Gl.1 (M87661)

c Position in the norovirus genomic sequence GII.1 (U07611)

${ }^{\mathrm{d}}$ Position in the norovirus genomic sequence Gll.4 (AY032605) 
A)

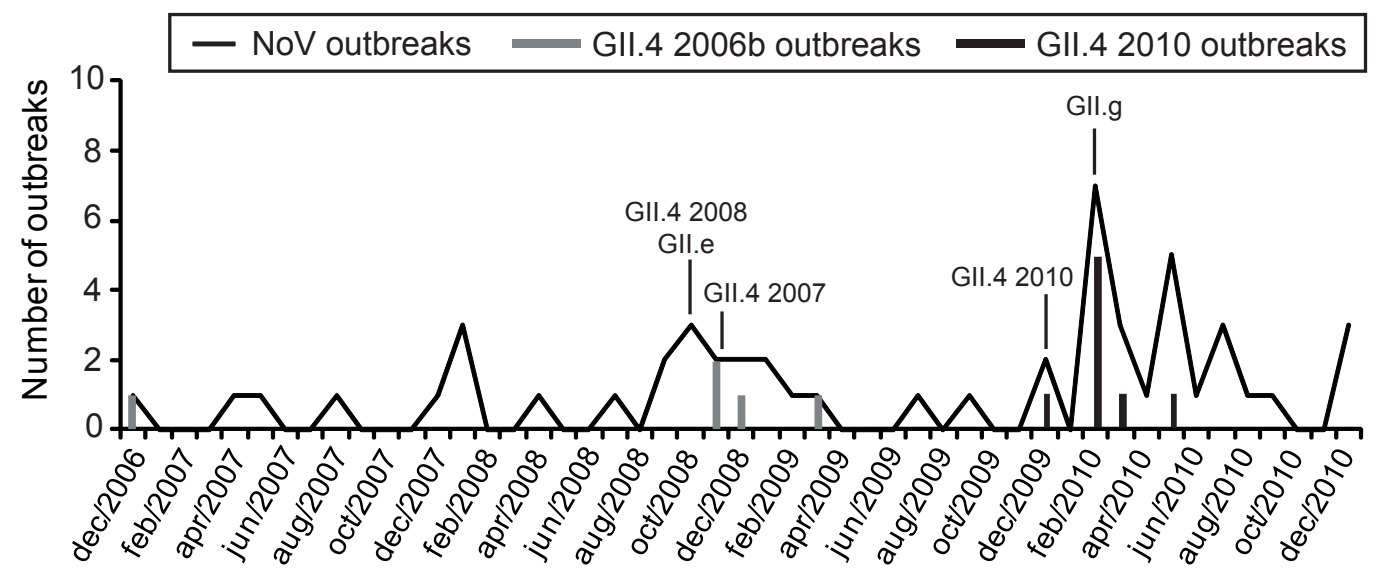

B)

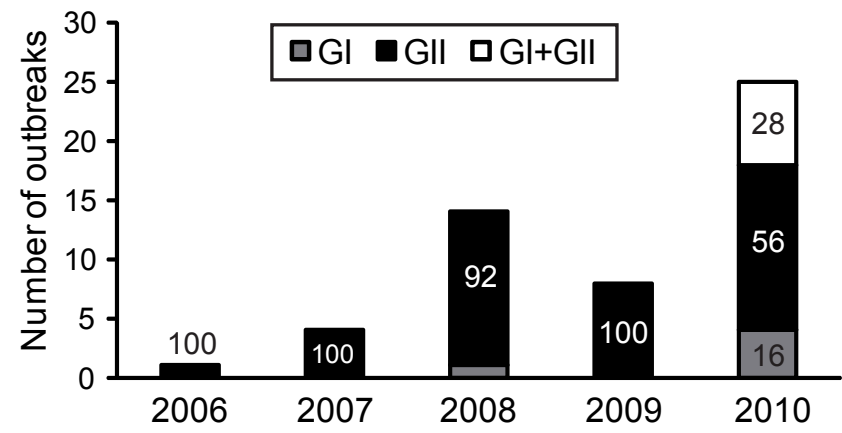

C)

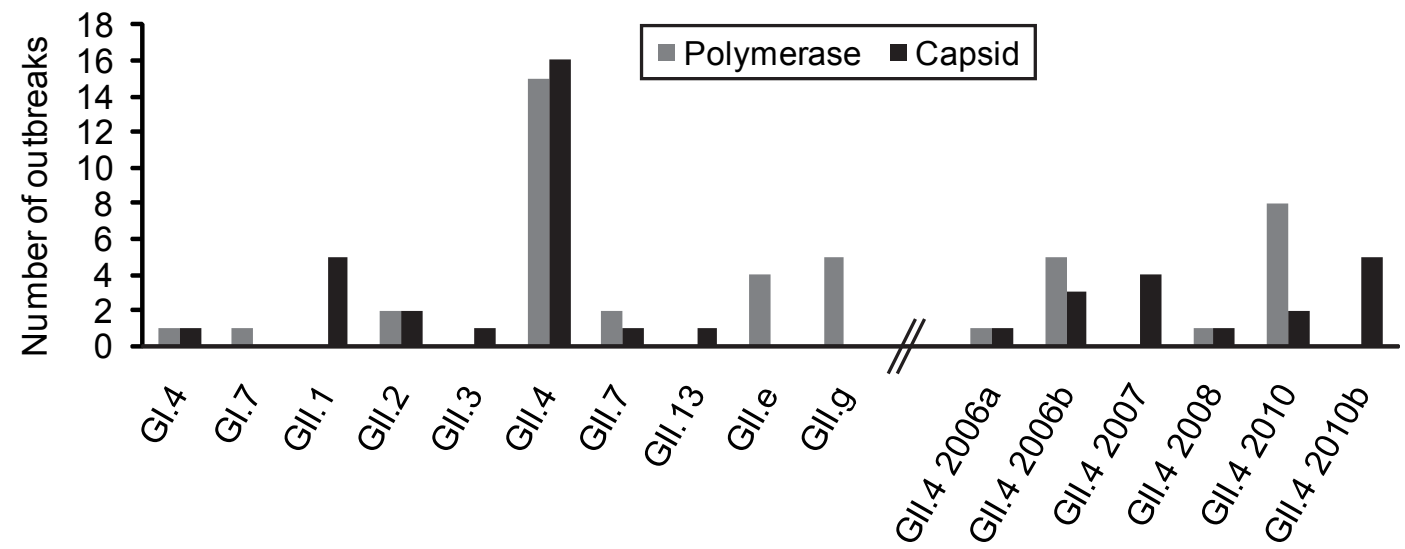

Figure 1 Characteristics of noroviruses detected in acute gastroenteritis outbreaks reported in Belgium. A) Monthly distribution of norovirus (NoV)-associated outbreaks in Belgium from December 2007 to December 2010. Months corresponding to the primer detection of novel NoV genotypes (GIl.e and Gll.g) or sub-lineages (Gll.4 variants 2007, 2008 and 2010) are indicated by an arrow; Diversity of NoV

genogroups (B), genotypes and Gll.4 sub-lineages (C) detected in gastro-enteritis outbreaks in Belgium during 2006 and 2010. Percentages of genogroup prevalence are indicated in the vertical bars. Typing results were obtained by phylogenetic clustering with reference strains (http://www.noronet.nl/) either for the partial polymerase gene sequence (grey) or the partial capsid gene sequence (black).

2010, GII NoVs outbreaks largely predominated and represented $90.4 \%$ of the NoVs either in single infections or in co-infection with GI NoVs (Figure 1B). GI single infections and GI-GII co-infections represented respectively 16 and $28 \%$ of the outbreaks reported in 2010. Phylogenetic analysis allowed GII NoVs clustering into 7 different genotypes: GII.4 strains (15 outbreaks,
50\%), recombinant GII.g/GII.1 (5 outbreaks, 16.7\%), recombinant GII.e/GII.4 (3 outbreaks, 10\%), GII.2 (2 outbreaks, 6.6\%), GII.7 (2 outbreaks, 6.6\%), GII.13 (1 outbreak, 3.3\%) and recombinant GII.e/GII.3 (1 outbreak, 3.3\%) (Figure 1C). Two GI NoV outbreaks were successfully genotyped and clustered into GI.4 and GI.7. Figure $1 \mathrm{C}$ also shows the variety of GII.4 variants 
identified since 2006: 2006a, 2006b, 2007, 2008 and 2010; of all the GII.4 variants the majority clustered with the new GII.4 2010 variant. The only outbreak reported in 2006 was linked with GII.4 2006b whereas GII.4 2006a was identified in the only typed outbreak of 2007. The 2006b variants were detected until March 2009 in co-circulation with GII.4 variants 2007 and 2008; subsequently they were displaced by the 2010 variant first described in December 2009 (Figure 1A).

\section{Detection of novel GII.4 variants}

Phylogenetic clustering with GII NoV reference strain sequences from the Noronet European genotyping tool (Additional file 1 Table S1) allowed the characterisation of NoV circulating during the period of this study. Phylogenetic trees were drawn for both the partial polymerase and capsid gene sequences (Figure 2 and 3) and genotyping results for the amplified region of each sample are shown in table 1. GII.4 NoV sequences were shown to group within 4 and 6 clusters for the polymerase and capsid genes respectively. Besides the pandemic variants GII.4 2006a and GII.4 2006b, GII.4 sequences clustered with newly reported GII.4 sequences; 2007 (for the capsid gene only), 2008 and 2010. One single outbreak in 2008 was caused by GII.4 2008 variant whereas GII.4 2007 capsid gene sequences were amplified from samples originating from 4 outbreaks in late 2008 and early 2009. In two of these outbreaks, the 2007 variant was detected with the 2006b variant either in the same sample (IPH2839) or in two samples from the same outbreak (IPH3060 and IPH3069). In a sample from a third outbreak, a GII.4 2006b polymerase was found in combination with a GII.4 2007 capsid gene sequence (IPH2700). A GI.7 NoV was found co-circulating with GII.4 2010 in samples originating from an identical outbreak. Since December 2009, GII.4 2010 was the only GII.4 variant detected. Phylogenetic analyses showed that all GII.4 2010 sequences amplified from the polymerase gene grouped into one single cluster whereas the corresponding partial capsid gene sequences formed two different genetic clusters. Of these capsids, only two (IPH161 and IPH1010) belonged to the cluster formed by the GII.4 2010 reference strain. Although highly related (97.2 and 98\% similarity between the 253 bp long partial capsid gene sequences), the other strains grouped into a novel GII.4 capsid variant that we called GII.4 2010b.

\section{Identification of a novel GII recombinant}

The identification of inconsistent genotype or sub-genotype clustering for the partial polymerase and capsid gene sequences for NoV strains (Figure 2 and 3) allowed the description of 5 types of recombinants involved with 14 outbreaks: GII.4 2010/GII.4 2010b (5 outbreaks), GII. g/GII.1 (4 outbreaks), GII.e/GII.4 2007 (3 outbreaks),
GII.e/GII.3 (1 outbreak) and GII.4 2006b/GII.4 2007 (1 outbreak). Except from the GII.4 intergenotype recombinants, recombinants identified in this study had GII polymerase genes for which no associated capsid genes have been described, namely GII.e and GII.g which shared $93.1-94.2 \%$ and $95.6-100 \%$ nucleotide identity with reference strains OC07138/07/JP and NSW199U/ 08/AU, respectively. GII.e polymerases were either detected in association with GII.4 2007 or GII.3 capsids (Figure 2; Figure 3; Figure 4C and 4D) whereas all GII.g polymerases were identified with GII.1 capsids (Figure 2; Figure 3 and 4B). Simplot analyses showed potential recombination cross-over for all the recombinant types to be located at, immediately upstream or downstream the ORF1-ORF2 overlap (Figure 4).

\section{Discussion}

During the monitoring of NoVs implicated with outbreaks in Belgium, the detection of novel GII recombinants and of GII.4 variants came along with increased outbreak reporting in 2008 and 2010. This is the first report of GII.e/GII.3 and GII.g/GII.1 recombinants implicated in outbreaks and molecular data obtained in this study for NoVs could be representative of the current epidemiological situation for Western European countries.

NoVs implicated in suspected foodborne outbreaks reported in Belgium between December 2006 and December 2010 emphasised their importance and implication in public health. Considering that, similarly to previously published data [35], the aetiology remains unknown for 20 to $50 \%$ of the reported outbreaks (data not shown), the implication of $\mathrm{NoV}$ in $11.8 \%$ of the outbreaks showed the importance of NoVs in foodborne outbreaks in Belgium. The presence of NoV in food was confirmed in 16 of the 35 (46\%) suspected foodborne outbreaks for which food samples were provided (data not shown). Unfortunately, no sequences could be obtained from these matrices. Also, epidemiological information never involved the consumption of primary infected foodstuffs in outbreaks caused by NoV [36]. Thus, outbreaks analysed in the study period mainly consisted in NoV outbreaks where human-to-human transmissions were involved either by the consumption of food contaminated by a foodhandler or by direct contact. Hence, NoVs detected from these outbreaks reflect the panel of NoVs circulating among individuals in a contemporaneous population.

As commonly reported in the literature, GII NoVs largely predominated (90.4\% of all outbreaks) and GII.4 genotype NoVs were identified in 16 of the 29 typed outbreaks (55.2\%). Phylogenetic analyses indicated that GII NoV strains detected in Belgium formed different clades and even subclusters in the pylogenetics trees, 


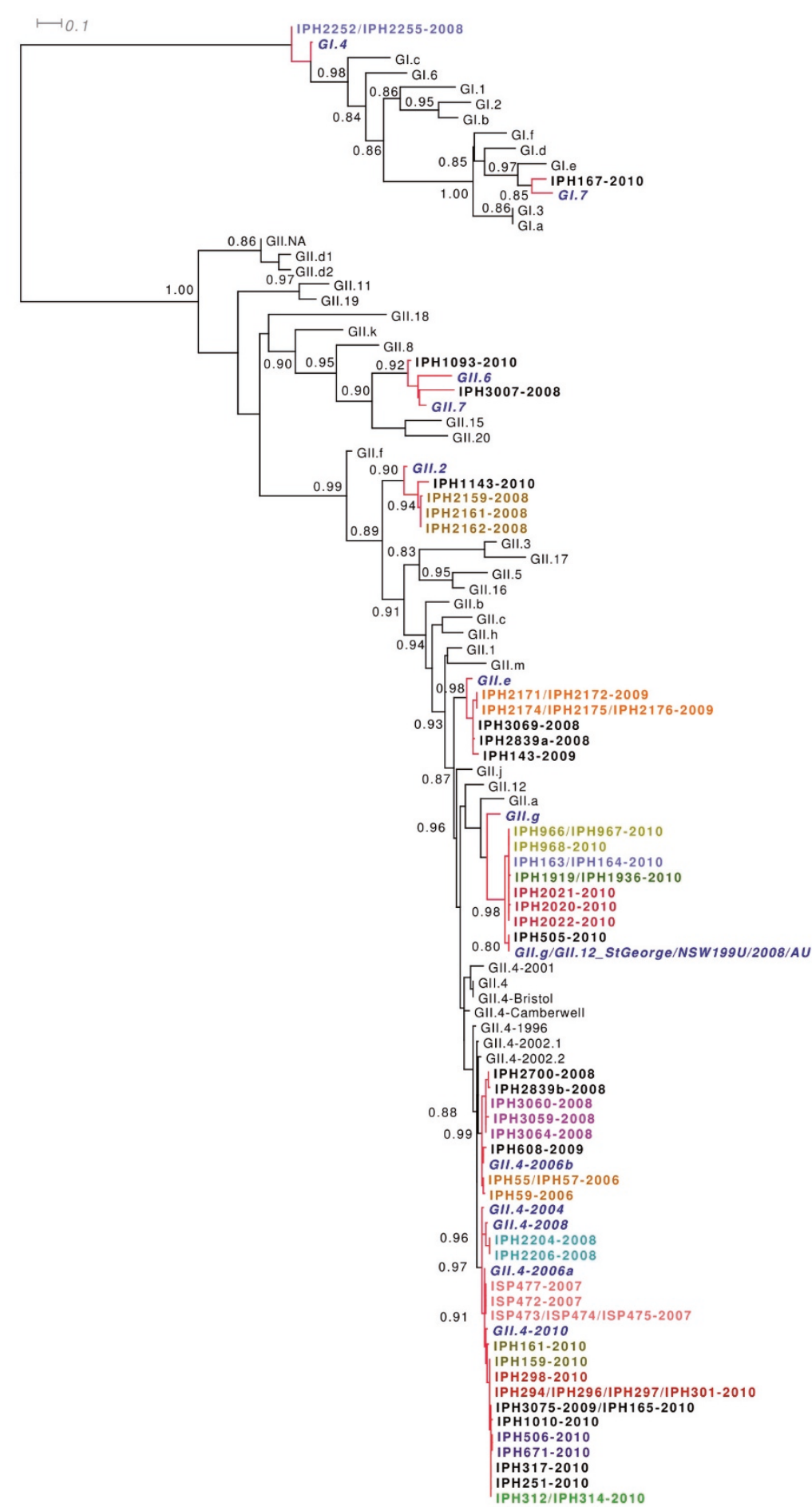

Figure 2 Phylogenetic analyses of the partial polymerase region of the detected norovirus genomes. Phylogenetic trees were inferred under a maximum-likelihood framework from the nucleic acid sequences aligned at the protein level (GTR model with aLRT node support, see Material and Methods for details). The aLRT node supports were only indicated when superior to 0.8 and relevant to the genotype identification. Reference NoV strains identified as highly related to the norovirus (NoV) samples were highlighted in bold, italic blue font. NoV samples originating from the same outbreak and co-localized in the phylogenetic tree were also highlighted in color. Identical NoV sequences were represented on the same node (in color if originating from the same outbreak, in black if originating from different outbreaks). Details on the genotyping of each NoV sample can be found in Table 1. 


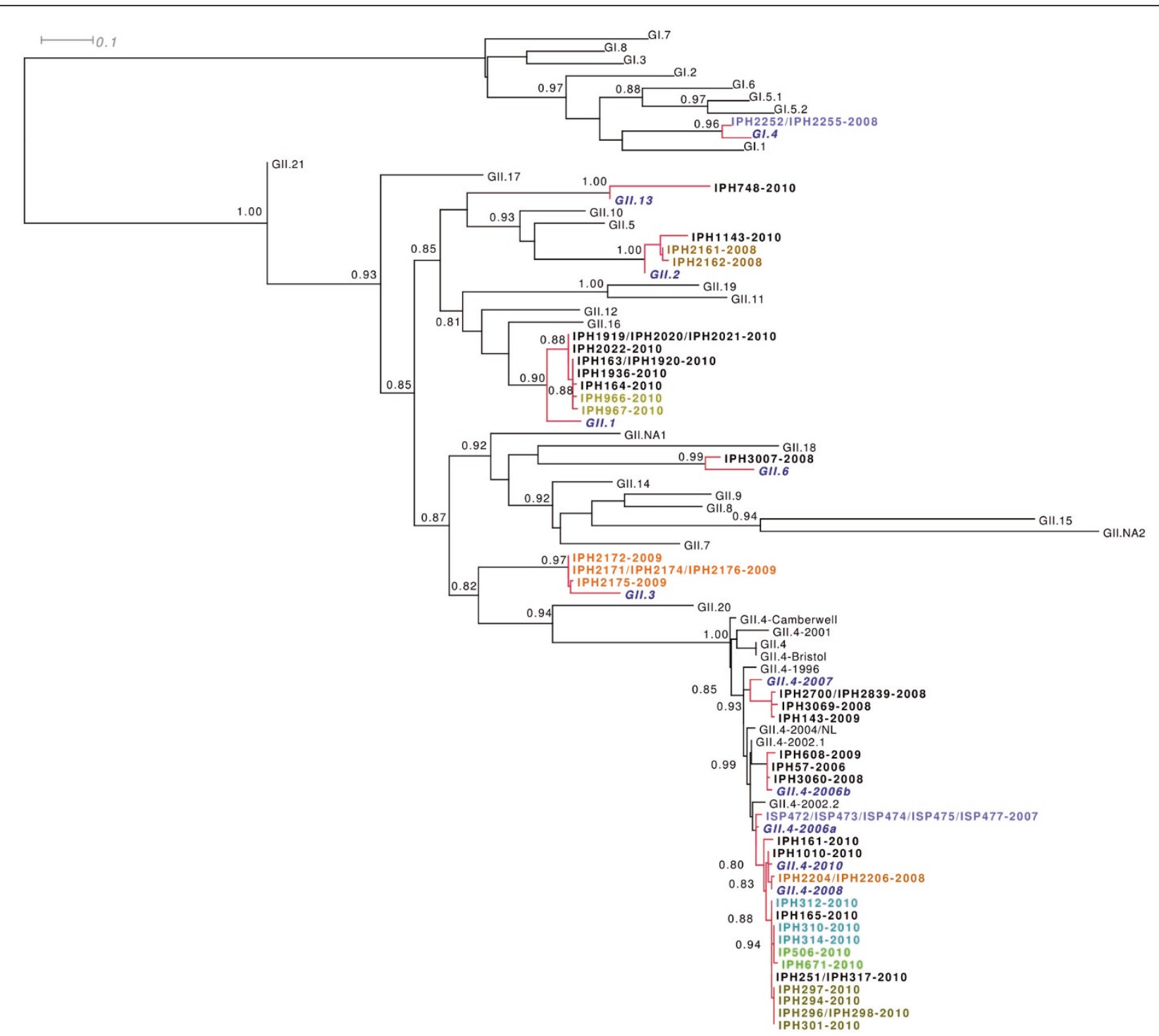

Figure 3 Phylogenetic analyses of the partial capsid region of the detected norovirus genomes. Phylogenetic trees were inferred under a maximum-likelihood framework from the nucleic acid sequences aligned at the protein level (GTR model with aLRT node support, see Material and Methods for details). The aLRT node supports were only indicated when superior to 0.8 and relevant to the genotype identification. Reference NoV strains identified as highly related to the norovirus (NoV) samples were highlighted in bold, italic blue font. NoV samples originating from the same outbreak and co-localized in the phylogenetic tree were also highlighted in color. Identical NoV sequences were represented on the same node (in color if originating from the same outbreak, in black if originating from different outbreaks). Details on the genotyping of each NoV sample can be found in Table 1.

especially for GII.4 NoVs (Figure 1C, 2 and 3). GII.4 subtyping identified the circulation of 5 different variants since the winter of 2006 namely 2006a, 2006b, 2007, 2008 and 2010. GII.4 2008 was identified in one single outbreak in 2008 and disappeared the same year. This GII.4 2008 variant has been associated at its primary description in Germany with a very severe case of gastroenteritis in a young boy [37]. Further data on the success of this sub-lineage in Europe and Asia are rather contradictory and report either discreet circulation
$[38,39]$ or stark implication $[40,41]$ in epidemics for similar study periods. GII.4 2006b variants were repeatedly reported until 2009 in co-circulation with variants 2007 and 2008 before being displaced by newly emerged GII.4 2010 variants the following year. Multiple reports showed that until 2009, GII.4 2006b variants predominated upon the newly emerged GII.4 2007 and 2008 variants in other countries $[38,42]$. The emergence of the GII.4 2008 sub-lineage did not lead to a larger disease burden during the following winter season suggesting 

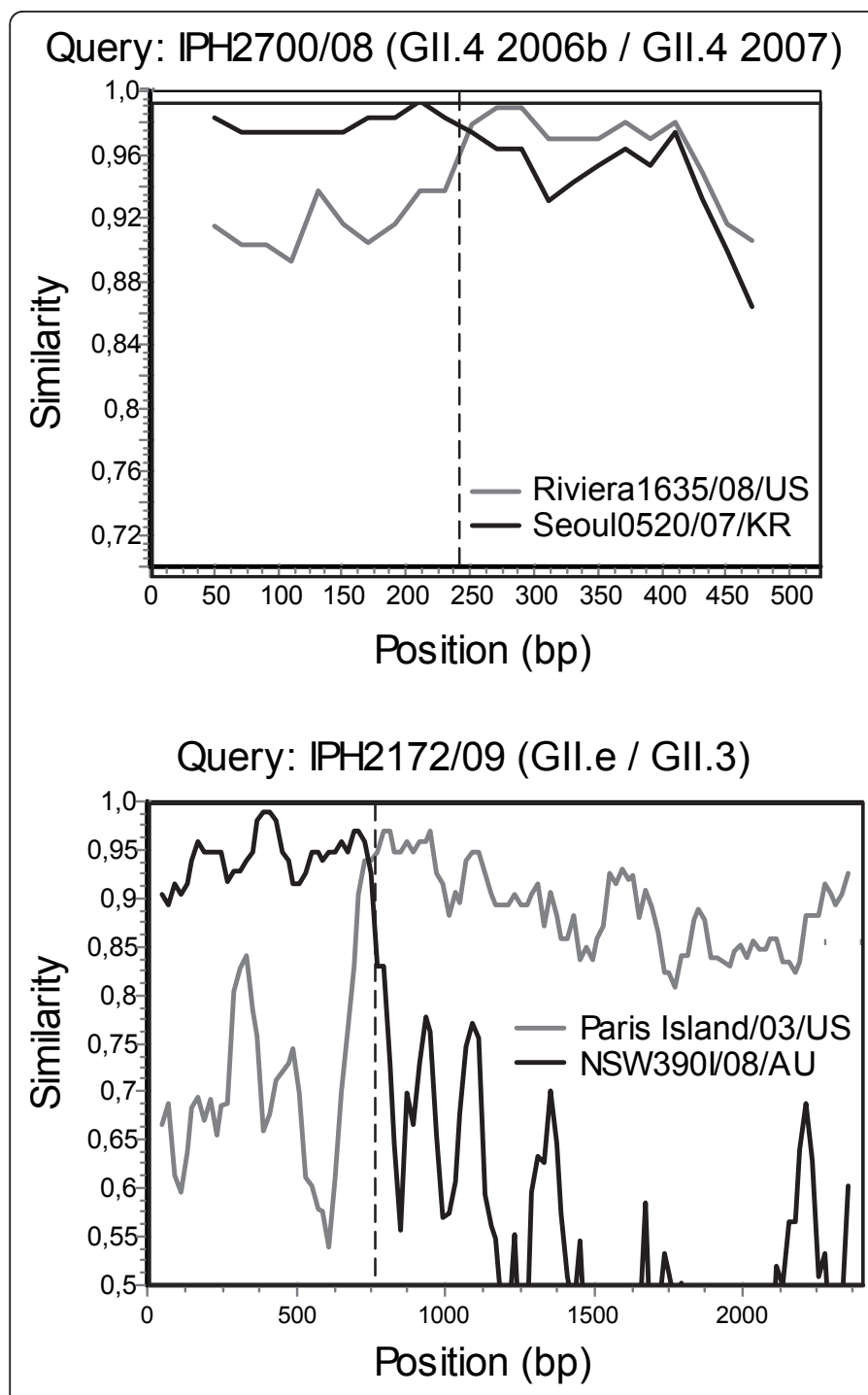

Query: IPH0163/10 (GII.g / GII.1)

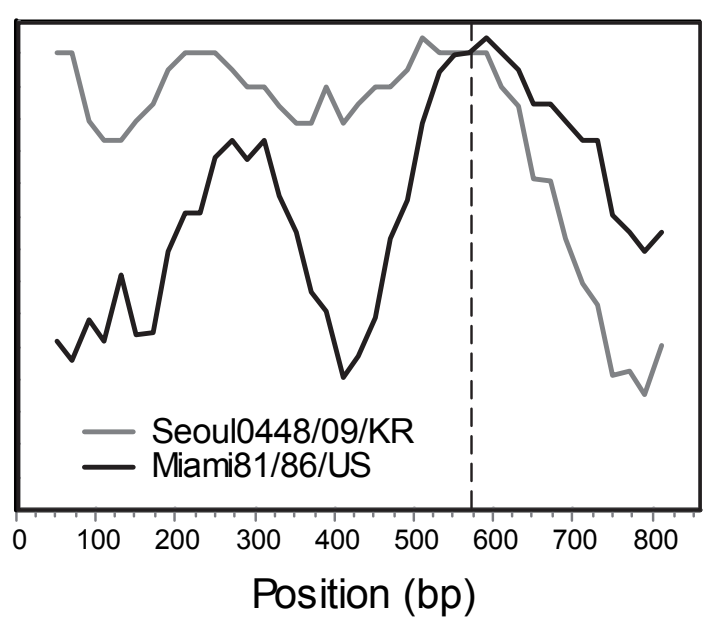

Query: IPH0143/09 (GII.e / GII.4 2007)

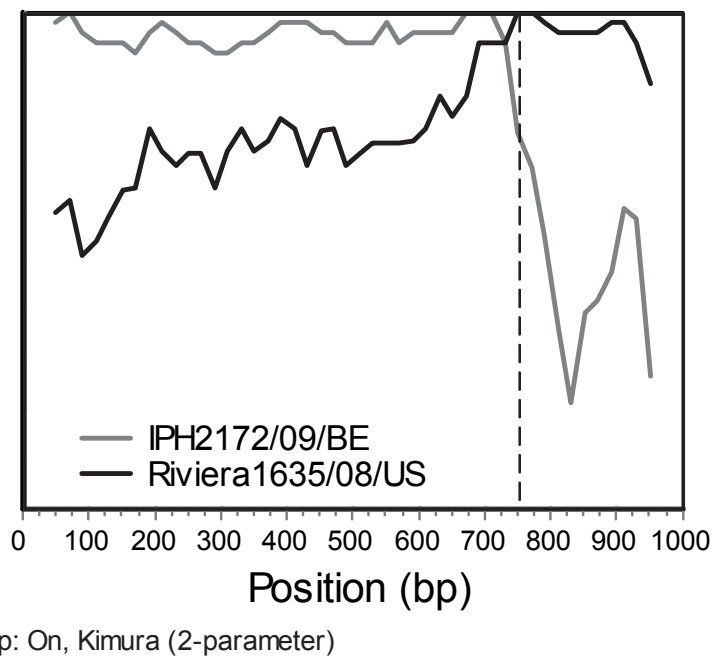

Figure 4 Similarity plots of norovirus GIl recombinants IPH2700/08 (A), IPH0163/10 (B), IPH2172/09 (C) and IPH0143/09 (D). SimPlot analyses were conducted with partial polymerase and capsid gene sequence alignments. Sequences, either a concatemer of partial polymerase and capsid sequences (A) or sequences covering the ORF1/2 overlap (B-D) were analyzed for recombination. Graphs show the similarity of the putative parental reference strains with the recombinant GII NoVs detected in our study relative to the genomic position (bp). Dashed vertical lines indicate the start of the capsid gene. Genbank accession numbers from parental NoV strains are as follows: Miami81/1986/US (GIl.1): AF414416; NSW390I/2008/AU (GIl.4 2007): GQ845369; Paris Island/2003/US (GIl.b/GIl.3): AY652979; Riviera1635/2008/US (GIl.4 1996/GIl.4 2007): GQ413969; Seoul0448/2009/KR (GIl.g/GIl.12): HM635104; Seoul0520/2007/KR (Gll.4 2006b): FJ913975.

that some GII.4 variants do not dispose of selective advantages allowing their massive spread and longer persistence. In Belgium, GII.4 2010 constituted the only GII.4 variant circulating in 2010 and was involved in 8/ 18 typed outbreaks reported in the winter 2009-2010. Since its first detection in December 2009, the GII.4 2010 variant seemed to have definitively displaced the 2006b sub-lineage suggesting a perfect candidate for the generation of the observed new burden in NoV epidemics in 2010. Highly similar GII.4 2010 variants were identified in France as early as February 2009 [40], and also in Asia, Germany and Hungary since 2008 (BLAST analyses, data not shown) indicating its dissemination might have been widespread. More surveillance data from other continents will be needed to determine the implication of the GII.4 2010 sub-lineage in global epidemics. In this 4-year study period a diversity of GII.4 subvariants was observed with the subsequent emergence of three GII.4 variants. Our results clearly support that GII.4 variants evolve more rapidly than any other 
genotypes as suggested by the reported 5 to 36 -fold higher mutation rate in GII.4 compared to non GII.4 strains [43].

NoV outbreaks were not exclusively linked with GII.4 viruses, 6 distinct genotypes were identified for both the polymerase (GII.g, GII.e, GII.2, GII.7, GI.4 and GI.7) and the capsid (GII.1, GII.2, GII.3, GII.7, GII.13 and GI.4) genes. Phylogenetic analysis of partial sequences of the polymerase and of the $\mathrm{N}$-terminal conserved region of the capsid protein resulted in trees with different topologies for 5 different NoV strains, suggesting that recombination had occurred within these viruses. Four novel GII intergenotype and intersub-genotype recombinants (GII.e/GII.3, GII.g/GII.1, GII.4 2006b/GII.4 2007 and GII.4 2010/GII.4 2010b) were detected along with 1 previously published recombinant (GII.e/GII.4 2007). Since the end of 2008, GII.e and GII.g polymerase gene sequences were amplified in samples from 9 outbreaks in association with GII.3 or GII.4 2007 and GII.1 capsid gene sequences, respectively. Previously published sequences covering the ORF1-ORF2 junction showed that similar polymerase gene sequences have been described exclusively in association with GII.4 2007 capsids for GII.e [44-46] and GII.12 capsids for GII.g $[41,44]$. The fact that GII.e and GII.g recombinants identified in this study harboured other capsids than previously described might indicate that they emerged after undergoing a recent recombination event. As previously described for GII.b recombinants, several different capsid sequences can be associated with the GII.e and GII.g polymerase genes [17]. The association of these polymerases with distinct capsid gene sequences could provide them some selective advantages over monophylogenic strains. Indeed, GII.e and GII.g were both the second most prevalent genotype after GII.4 in 2008-2009 and 2010, respectively. These observations suggest that the emergence of the GII.e and GII.g recombinant NoVs could partly explain the increase in NoV activity observed for these periods. Data upon the NoVs implicated with the latest NoV epidemics across the world will be needed to confirm the importance of these polymerases in NoV epidemiology. The origin of these polymerases, however, still remains unclear as no parental full-length sequences yet have been detected.

An intersub-genotype GII.4 2006a/GII.4 2007 recombinant was described based upon a concatemer of partial polymerase and capsid gene sequences. Direct sequencing did not show any sign of co-infection but in the absence of a sequence covering the ORF1-ORF2 junction, this could not be ruled out. GII.4 2006b and GII.e/ GII.4 2007 were shown to co-circulate in some 2008 outbreaks offering an opportunity for recombination in case of a multiple NoV infection. A recent study showed that part of the GII.4 genotypes circulating in Japan were in fact mosaics of former GII.4 sub-lineages [46]. Consequently, genetic recombination, in combination with small-scale mutations, is believed to play a role in the divergent evolution of GII.4 NoVs. A second potential intersub-lineage recombinant was identified based upon discordant tree topologies between the partial polymerase and capsid gene sequences of some GII.4 2010 variants. Indeed, in these samples all polymerase sequences clustered into one single group whereas the capsid gene sequences grouped into 2 distinct clusters. In the absence of a reference strain for the partial capsid gene sequences that did not cluster with GII.4 2010, they were referred to as GII.4 2010b. Although, sequencing of whole capsid genes would allow accurate characterisation of this novel variant. In this study, the highly conserved region $\mathrm{C}$ in the capsid gene was amplified for nearly all strains and only few full capsid gene sequences were successfully amplified. Amplifying long sequences from clinical samples remains challenging and in this study efforts were focused on the obtaining of sequences covering the ORF1-ORF2 overlap for the study of recombination breakpoints. SimPlot analyses confirmed all recombination points to be located at this junction which corresponds to the previously proposed hotspot for NoV recombination $[21,47]$.

The nomenclature used in our study is based upon the clustering with $\mathrm{NoV}$ reference strains given for the $\mathrm{NoV}$ genotyping tool of the Dutch National Institute of Public health and the Environment made available by Noronet [48]. Great discrepancy is observed for NoV genotypes in the literature in particular for GII.4 sublineages that are commonly named after the year of detection. For instance, NoV strains related to the GII.4 2007 variants detected in this study were referred to as Cairo \& Osaka 2007 in France and Australia [40,44], GII.4 2007a in Japan [46], GII.4 2008a in Canada [49] and cluster $C$ in India [15]. Therefore, a common language still needs to be adopted in order to compare data between research teams. We have chosen reference strains provided by Noronet because one of the aims of the Noronet network is the elaboration of a well founded standardized nomenclature for NoVs.

\section{Conclusions}

Although highly diverse, NoVs circulating in Belgium in the past 4 years were predominantly GII.4 sub-lineages and GII NoV recombinants indicating that both the accumulation of mutations and genetic recombination constitute major driven forces in NoV evolution. Furthermore, the emergent new GII.4 variants or GII recombinants might have had an impact on the magnitude of $\mathrm{NoV}$ epidemics suggesting that these strains dispose of some selective advantages over other circulating NoV strains. 
A comprehensive study of the NoVs strains predominantly and contemporaneously involved in large and cost-effective gastro-enteritis outbreaks would help targeting the development of vaccines, therapeutic strategies and/or preventive measure on these particular strains.

\section{Additional material}

Additional file 1: Table 1 - Reference sequences used for phylogenetic analysis (http://www.noronet.nl).

Additional file 2: Table 2 - Food-borne gastroenteritis outbreaks reported in Belgium between 2006 and 2010

\section{Acknowledgements}

We thank the staff of the Virology Service (Ulg) and the Food Pathogen Service (IPH) for the technical assistance, especially Dominique Ziant, Thomas Delwiche, Elke Wattijn and Donia Baccari. This study was supported by grants from the Belgian Science Policy 'Science for a Sustainable Development' (SD/AF/01 and SD/CL/09) and the University of Liège 'Fonds speciaux pour la Recherche-crédits classiques' 2008-2009 (C-09/60).

\section{Author details}

'Department of Infectious and Parasitic Diseases, Veterinary Virology and Animal Viral Diseases, Faculty of Veterinary Medicine, University of Liege, Liege, Belgium. ${ }^{2}$ Department of Infectious and Parasitic Diseases, Immunology and Vaccinology, Faculty of Veterinary Medicine, University of Liege, Liege, Belgium. ${ }^{3}$ Communicable and Infectious Diseases, Food borne Pathogens, Scientific Institute of Public Health, Brussels, Belgium.

\section{Authors' contributions}

EM together with AS performed the molecular biology on NoV-positive faecal samples for sequencing, genotyping and bioinformatics. EM drafted the manuscript. SD and NB jointly collected clinical samples from outbreaks, gathered information about the outbreaks and detected NoV presence by performing RT-QPCR. LP performed all bioinformatic analyses (alignments, phylogeny and recombination studies). AV, ET and KD were responsible for the supervision of the study. All authors read and approved the final manuscript.

\section{Competing interests}

The authors declare that they have no competing interests.

Received: 2 March 2011 Accepted: 18 June 2011

Published: 18 June 2011

\section{References}

1. Siebenga J, Duizer E, Koopmans M: Norovirus Epidemiology. In Caliciviruses: Molecular and Cellular Virology. Edited by: Hansman GS, Jiang XJ, Green KY. Norfolk: Caister Academic Press; 2010:1-24.

2. Lopman BA, Reacher MH, Van Duijnhoven $Y$, Hanon FX, Brown D, Koopmans M: Viral gastroenteritis outbreaks in Europe, 1995-2000. Emerg Infect Dis 2003, 9:90-96.

3. Leuenberger S, Widdowson M, Feilchenfeldt J, Egger R, Streuli R: Norovirus outbreak in a district general hospital - new strain identified. Swiss Med Wkly 2007, 137:57-81.

4. Koopmans M: Noroviruses in healthcare settings: a challenging problem. J Hosp Infect 2009, 73:331-337.

5. Green KY, Ando T, Balayan MS, Berke T, Clarke IN, Estes MK, Matson DO, Nakata S, Neill JD, Studdert MJ, Thiel HJ: Taxonomy of the caliciviruses. $J$ Infect Dis 2000, 181:S322-330.

6. Sosnovtsev SV, Belliot G, Chang KO, Prikhodko VG, Thackray LB, Wobus CE, Karst SM, Virgin HW, Green KY: Cleavage map and proteolytic processing of the murine norovirus nonstructural polyprotein in infected cells. J Virol 2006, 80:7816-7831.
7. Vennema H, de Bruin E, Koopmans M: Rational optimization of generic primers used for Norwalk-like virus detection by reverse transcriptase polymerase chain reaction. J Clin Virol 2002, 25:233-235.

8. Bertolotti-Ciarlet A, Crawford SE, Hutson AM, Estes MK: The 3' end of Norwalk virus mRNA contains determinants that regulate the expression and stability of the viral capsid protein VP1: a novel function for the VP2 protein. J Virol 2003, 77:11603-11615.

9. Ando T, Noel JS, Fankhauser RL: Genetic classification of "Norwalk-like viruses. J Infect Dis 2000, 181:S336-348.

10. Zheng DP, Ando T, Fankhauser RL, Beard RS, Glass Rl, Monroe SS: Norovirus classification and proposed strain nomenclature. Virology 2006, 346:312-323.

11. Kageyama T, Shinohara M, Uchida K, Fukushi S, Hoshino FB, Kojima S, Takai R, Oka T, Takeda N, Katayama K: Coexistence of multiple genotypes, including newly identified genotypes, in outbreaks of gastroenteritis due to Norovirus in Japan. J Clin Microbiol 2004, 42:2988-2995.

12. Kroneman A, Harris J, Vennema H, Duizer E, van Duynhoven Y, Gray J, Iturriza M, Bottiger B, Falkenhorst $\mathrm{G}$, Johnsen $\mathrm{C}$, von Bonsdorff $\mathrm{CH}$ Maunula L, Kuusi M, Pothier P, Gallay A, Schreier E, Koch J, Szucs G, Reuter G, Krisztalovics K, Lynch M, McKeown P, Foley B, Coughlan S, Ruggeri FM, Di Bartolo I, Vainio K, Isakbaeva E, Poljsak-Prijatelj M, Grom AH, et al: Data quality of 5 years of central norovirus outbreak reporting in the European Network for food-borne viruses. J Public Health 2008, 30:82-90.

13. Lopman B, Vennema H, Kohli E, Pothier P, Sanchez A, Negredo A, Buesa J, Schreier E, Reacher M, Brown D, Gray J, Iturriza M, Gallimore C, Bottiger B, Hedlund K-O, Torvén M, von Bonsdorff C-H, Maunula L, Poljsak-Prijatelj M, Zimsek J, Reuter G, Szücs G, Melegh B, Svennson L, van Duijnhoven Y, Koopmans M: Increase in viral gastroenteritis outbreaks in Europe and epidemic spread of new norovirus variant. The Lancet 2004, 363:682-688

14. Bull RA, Tu ET, Mclver CJ, Rawlinson WD, White PA: Emergence of a new norovirus genotype II.4 variant associated with global outbreaks of gastroenteritis. J Clin Microbiol 2006, 44:327-333.

15. Nayak MK, Chatterjee D, Nataraju SM, Pativada M, Mitra U, Chatterjee MK, Saha TK, Sarkar U, Krishnan T: A new variant of Norovirus Gll.4/2007 and inter-genotype recombinant strains of NVGIl causing acute watery diarrhoea among children in Kolkata, India. J Clin Virol 2009, 45:223-229.

16. Bruggink LD, Marshall JA: Molecular and epidemiological features of Glllb norovirus outbreaks in Victoria, Australia, 2002-2005. J Med Virol 2009, 81:1652-1660.

17. Reuter G, Vennema H, Koopmans M, Szucs G: Epidemic spread of recombinant noroviruses with four capsid types in Hungary. I Clin Virol 2006, 35:84-88.

18. Martella V, Decaro N, Lorusso E, Radogna A, Moschidou P, Amorisco F, Lucente MS, Desario C, Mari V, Elia G, Banyai K, Carmichael LE, Buonavoglia C: Genetic heterogeneity and recombination in canine noroviruses. J Virol 2009, 83:11391-11396.

19. Mauroy A, Scipioni A, Mathijs E, Thys C, Thiry E: Molecular detection of kobuviruses and recombinant noroviruses in cattle in continental Europe. Arch Virol 2009, 154:1841-1845.

20. Muller B, Klemm U, Mas Marques A, Schreier E: Genetic diversity and recombination of murine noroviruses in immunocompromised mice. Arch Virol 2007, 152:1709-1719.

21. Bull RA, White PA: Genome Organization and Recombination. In Caliciviruses Molecular and Cellular Virology. Edited by: Hansman GS, Jiang XJ, Green KY. Norfolk: Caister Academic Press; 2010:45-63.

22. Baert L, Uyttendaele M, Debevere J: Evaluation of viral extraction methods on a broad range of Ready-To-Eat foods with conventional and realtime RT-PCR for Norovirus Gll detection. Int J Food Microbiol 2008, 123:101-108.

23. Loisy F, Atmar RL, Guillon P, Cann Pl, Pommepuy M, Guyader FSI: Real-time RT-PCR for norovirus screening in shellfish. J Virol Methods 2005, 123:1-7.

24. Svraka S, Duizer E, Vennema $H$, de Bruin $E$, van der Veer B, Dorresteijn B, Koopmans M: Etiological role of viruses in outbreaks of acute gastroenteritis in The Netherlands from 1994 through 2005. J Clin Microbiol 2007, 45:1389-1394.

25. Stals A, Baert L, Botteldoorn N, Werbrouck H, Herman L, Uyttendaele M, Van Coillie E: Multiplex real-time RT-PCR for simultaneous detection of GI/GII noroviruses and murine norovirus 1. J Virol Methods 2009, 161:247-253. 
26. Kojima S, Kageyama T, Fukushi S, Hoshino FB, Shinohara M, Uchida K, Natori K, Takeda N, Katayama K: Genogroup-specific PCR primers for detection of Norwalk-like viruses. J Virol Methods 2002, 100:107-114.

27. Kamel AH, Ali MA, El-Nady HG, de Rougemont A, Pothier P, Belliot G: Predominance and circulation of enteric viruses in the region of Greater Cairo, Egypt. J Clin Microbiol 2009, 47:1037-1045

28. Edgar RC: MUSCLE: multiple sequence alignment with high accuracy and high throughput. Nucleic Acids Res 2004, 32:1792-1797.

29. Guindon S, Gascuel O: A simple, fast, and accurate algorithm to estimate large phylogenies by maximum likelihood. Syst Biol 2003, 52:696-704.

30. Tavaré S: Some Probabilistic and Statistical Problems in the Analysis of DNA sequences. Lectures Math Life Sci 1986, 17:57-86.

31. Yang Z: Maximum likelihood phylogenetic estimation from DNA sequences with variable rates over sites: approximate methods. J Mol Evol 1994, 39:306-314.

32. Anisimova M, Gascuel O: Approximate likelihood-ratio test for branches: A fast, accurate, and powerful alternative. Syst Biol 2006, 55:539-552.

33. Hordijk W, Gascuel O: Improving the efficiency of SPR moves in phylogenetic tree search methods based on maximum likelihood. Bioinformatics 2005, 21:4338-4347.

34. Guindon S, Delsuc F, Dufayard JF, Gascuel O: Estimating maximum likelihood phylogenies with PhyML. Methods Mol Biol 2009, 537:113-137.

35. Olsen SJ, MacKinnon LC, Goulding JS, Bean NH, Slutsker L: Surveillance for Foodborne-Disease Outbreaks - United States, 1993-1997. Morb Mortal Wkly Rep 2000, 49:1-65.

36. Baert L, Uyttendaele M, Stals A, Van Coillie E, Dierick K, Debevere J, Botteldoorn N: Reported foodborne outbreaks due to noroviruses in Belgium: the link between food and patient investigations in an international context. Epidemiol Infect 2009, 137:316-325.

37. Schenk S, Petzold A, Hoehne M, Adam R, Schroten H, Tenenbaum T: Severe gastroenteritis with secondary fever in a 10-month-old boy. J Clin Virol 2010, 47:107-109.

38. Iritani N, Kaida A, Kubo H, Abe N, Goto K, Ogura H, Seto Y: Molecular epidemiology of noroviruses detected in seasonal outbreaks of acute nonbacterial gastroenteritis in Osaka City, Japan, from 1996-1997 to 2008-2009. J Med Virol 2010, 82:2097-2105.

39. Iritani N, Vennema H, Siebenga JJ, Siezen RJ, Renckens B, Seto Y, Kaida A, Koopmans M: Genetic analysis of the capsid gene of genotype GIl.2 noroviruses. J Virol 2008, 82:7336-7345.

40. Belliot G, Kamel AH, Estienney M, Ambert-Balay K, Pothier P: Evidence of emergence of new GGIl.4 norovirus variants from gastroenteritis outbreak survey in France during the 2007-to-2008 and 2008-to-2009 winter seasons. J Clin Microbiol 2010, 48:994-998.

41. Han TH, Kim CH, Chung JY, Park SH, Hwang ES: Emergence of norovirus GII-4/2008 variant and recombinant strains in Seoul, Korea. Arch Virol 2011, 156:323-329.

42. Kremer JR, Langlet J, Skraber S, Weicherding P, Weber B, Cauchie HM, De Landtsheer S, Even J, Muller CP, Hoffmann L, Mossong J: Genetic diversity of noroviruses from outbreaks, sporadic cases and wastewater in Luxembourg 2008-2009. Clin Microbiol Infect 2010

43. Bull RA, Hyde J, Mackenzie JM, Hansman GS, Oka T, Takeda N, White PA Comparison of the replication properties of murine and human calicivirus RNA-dependent RNA polymerases. Virus Genes 2010, 42:16-27.

44. Eden J-S, Bull RA, Tu E, Mclver CJ, Lyon MJ, Marshall JA, Smith DW, Musto J, Rawlinson WD, White PA: Norovirus Gll.4 variant 2006b caused epidemics of acute gastroenteritis in Australia during 2007 and 2008. J Clin Virol 2010, 49:265-271.

45. Westrell T, Dusch V, Ethelberg S, Harris J, Hjertqvist M, Jourdan-da Silva N, Koller A, Lenglet A, Lisby M, Vold L: Norovirus outbreaks linked to oyster consumption in the United Kingdom, Norway, France, Sweden and Denmark, 2010. Euro Surveill 2010, 15: pii = 19524[http://www. eurosurveillance.org/ViewArticle.aspx?Articleld = 19524.].

46. Motomura K, Yokoyama M, Ode H, Nakamura H, Mori H, Kanda T, Oka T, Katayama K, Noda M, Tanaka T, Takeda N, Sato H: Divergent evolution of norovirus GII/4 by genome recombination from May 2006 to February 2009 in Japan. J Virol 2010, 84:8085-8097.

47. Mathijs E, Muylkens B, Mauroy A, Ziant D, Delwiche T, Thiry E: Experimental Evidence of Recombination in Murine Noroviruses. J Gen Virol 2010, 91:2723-2733.

48. National Institute of Public health and the Environment The Netherlands: Norovirus genotyping tool.[http://www.rivm.nl].
49. Pang XL, Preiksaitis JK, Wong S, Li V, Lee BE: Influence of novel norovirus Gll.4 variants on gastroenteritis outbreak dynamics in Alberta and the Northern Territories, Canada between 2000 and 2008. PLoS One 2010, 5 e11599.

50. Jiang X, Huang PW, Zhong WM, Farkas T, Cubitt DW, Matson DO: Design and evaluation of a primer pair that detects both Norwalk- and Sapporo-like caliciviruses by RT-PCR. J Virol Methods 1999, 83:145-154.

doi:10.1186/1743-422X-8-310

Cite this article as: Mathijs et al:: Novel norovirus recombinants and Gll.4 sub-lineages associated with outbreaks between 2006 and 2010 in Belgium. Virology Journal 2011 8:310.

\section{Submit your next manuscript to BioMed Central and take full advantage of:}

- Convenient online submission

- Thorough peer review

- No space constraints or color figure charges

- Immediate publication on acceptance

- Inclusion in PubMed, CAS, Scopus and Google Scholar

- Research which is freely available for redistribution
Ciomed Central 